\title{
Fast-Expanding Hi Shells Associated with Supernova Remnants in the I-GALFA Survey
}

\author{
Geumsook Park ${ }^{1}$, Bon-Chul Koo ${ }^{1}$, Steven J. Gibson ${ }^{2}$, \\ and Ji-hyun Kang ${ }^{3,4}$ \\ ${ }^{1}$ Department of Physics and Astronomy, Seoul National University, 1 Gwanak-ro, Gwanak-gu, \\ Seoul 151-742, Republic of Korea \\ email: pgs@astro.snu.ac.kr, koo@astro.snu.ac.kr \\ ${ }^{2}$ Department of Physics and Astronomy, Western Kentucky University, Bowling Green, KY \\ 42101, USA \\ email: steven.gibson@wku.edu \\ ${ }^{3}$ Yonsei University Observatory, Yonsei University, 50 Yonsei-ro, Seodaemun-gu, Seoul \\ 120-749, Republic of Korea \\ ${ }^{4}$ Korea Astronomy and Space Science Institute, 776 Daedeokdae-ro, Yuseong-gu, Daejeon \\ 305-348, Republic of Korea \\ email: jkang@kasi.re.kr
}

\begin{abstract}
We examine excess emission at high positive and negative velocities toward known Galactic supernova remnants (SNRs) in the "Inner-Galaxy Arecibo L-band Feed Array (IGALFA)" Hi 21-cm survey data. The I-GALFA survey covers $\ell=32^{\circ}$ to $77^{\circ}$, and has a velocity range of $\pm 700 \mathrm{~km} \mathrm{~s}^{-1}$ with high angular and velocity resolutions $\left(4^{\prime}\right.$ and $0.18 \mathrm{~km} \mathrm{~s}^{-1}$, respectively) and good sensitivity $(0.2 \mathrm{~K})$. The excess emission which is thought to be part of a fast-expanding Hi shell of a SNR is detected from four among 39 SNRs in the I-GALFA area: W44, G54.4-0.3, W51C, and CTB 80. Although the Hi shells of the four SNRs were already reported in low-resolution studies, the first detection of both sides of an expanding Hi shell associated with W44 is very inspiring. We discuss physical properties of these four SNRs and their statistical nature.
\end{abstract}

Keywords. ISM: supernova remnants, radio lines: ISM

\section{Introduction}

Supernova (SN) explosion is one of important sources to supply energy and matter to the interstellar medium (ISM). In the process, the ISM is swept up by supersonic shock waves, and produce expanding shells. The expanding shells are initially ionized, and over time cool down and neutralize. The neutral shells can be observed in Hi emission line at $21 \mathrm{~cm}$, but the identification is difficult because of Galactic foreground and background Hi emission. Nevertheless, shells with a large expanding velocity, larger than the maximum velocity allowed by the Galactic rotation in that direction, can be detected.

The I-GALFA survey observed the sky of $\ell=32^{\circ}$ to $77^{\circ}$ and $b \lesssim 10^{\circ}$ with high resolution $\left(4^{\prime}\right)$ and high sensitivity $(0.2 \mathrm{~K}$ ) (See Peek et al. 2010, 2011 for more details about the survey.). There are known 275 SNRs in our Galaxy (Green 2009a,b), and 39 among them are located in the I-GALFA area. We have done a systematic study of the Hi shells toward the Galactic SNRs using the I-GALFA data. 


\section{Results and Discussion}

We detected fast-moving Hi emission toward 4 of 39 SNRs in the I-GALFA area at high positive and/or negative velocities: G34.7 - 0.4 (W44), G49.2 - 0.7 (W51C), G54.4 - 0.3 (HC40), and G69.0 + 2.7 (CTB 80). The excess emission is well constrained within each $\mathrm{SNR}$ area in radio continuum, so it is thought that the emission is part of an atomic expanding shell of each SNR. The presence of high-velocity Hi shells in these SNRs was reported before by previous studies (e.g., Koo \& Heiles 1991, Koo et al. 1990, Koo \& Heiles 1995, Koo \& Moon 1997a), but it is the first time to observe both sides of an expanding shell associated with an SNR (W44) at high negative and positive velocities in Hi line. Also, in G54.4 - 0.3, the high-resolution I-GALFA map reveals a well-defined circular symmetric shell at high positive velocities.

We have derived physical parameters, such as an expansion velocity, ambient density, SN energy, age, and so on, by fitting the average Hi profiles from two SNRs, W44 and G54.4 - 0.3. The other two SNRs had been studied well in previous researches (Koo \& Moon 1997a, Koo et al. 1990, Koo \& Moon 1997b), and we adopt their parameters which are consistent with the I-GALFA results. The expansion velocities of the Hi shells range from 59 to $135 \mathrm{~km} \mathrm{~s}^{-1}$, and the ages of the SNRs are $1.8-9.5 \times 10^{4}$ yrs. Interestingly, all four SNRs with fast-expanding Hi shells (Hi SNRs) are all middle-aged SNRs, and their derived ambient densities $\left(0.8-400 \mathrm{~cm}^{-3}\right)$ are higher than that of warm $\left(\sim 0.1 \mathrm{~cm}^{-3}\right)$ or hot $\left(\sim 10^{-3} \mathrm{~cm}^{-3}\right)$ ISM. We suggest that the SNRs are probably core-collapse SN origin interacting with relatively dense medium.

To explore the fact that only a few of known SNRs in the I-GALFA area are detected as Hi SNRs, we examined statistics of SNRs with respect to a model showing the visibility of fast expanding Hi shells in the Galactic plane. There are many SNRs where Hi shells have not been detected in spite of their favorable locations, i.e., galactic longitudes that require relatively small velocities for expanding shells to be detected. These SNRs might be either too young or too old to have fast-expanding radiative Hi shells.

\section{References}

Green, D. A. 2009a, Bulletin of the Astronomical Society of India, 37, 45

Green, D. A. 2009b MNRAS, 399, 177

Koo, B.-C. \& Heiles, C. 1991, ApJ, 382, 204

Koo, B.-C. \& Heiles, C. 1995, ApJ, 442, 679

Koo, B.-C. \& Moon, D.-S. 1997a, ApJ, 475, 194

Koo, B.-C. \& Moon, D.-S. 1997b, ApJ, 485, 263

Koo, B.-C., Reach, W. T., Heiles, C., Fesen, R. A., \& Shull, J. M. 1990, ApJ, 364, 178

Peek, J. E. G., Begum, A., Douglas, K. A., et al. 2010, Astronomical Society of the Pacific Conference Series, 438, 393

Peek, J. E. G., Heiles, C., Douglas, K. A., et al. 2011, ApJS, 194, 20 